\title{
Direct probing of the free energy penalty for helix reversals and chiral mismatches in chiral supramolecular polymers
}

\author{
Benjamin Jouvelet ${ }^{l}$, Benjamin Isare ${ }^{l}$, Laurent Bouteiller $^{I^{*}}$ and Paul van der Schoot ${ }^{2}$ \\ ${ }^{1}$ UPMC Univ Paris 06, UMR 7610, Chimie des Polymères, F-75005 Paris, France, and CNRS, UMR \\ 7610, Chimie des Polymères, F-75005 Paris, France \\ ${ }^{2}$ Group Theory of Polymers and Soft Matter, Technische Universiteit Eindhoven, P.O. Box 513, 5600 \\ MB Eindhoven, The Netherlands, and Instituut voor Theoretische Fysica, Universiteit Utrecht, \\ Leuvenlaan 4, 3584 CE Utrecht, The Netherlands
}

laurent.bouteiller@upmc.fr

\begin{abstract}
Amplification of chirality, where a small imbalance in a chiral constituent is propagated into a strong optical purity, can occur in the spontaneous formation of helical 1-D stacks of molecules, stabilized, e.g., by hydrogen bonding, also known as supramolecular polymers. We have extended a statistical model by van Gestel et al., describing the highly non-linear relation between supramolecular helicity and enantiomeric excess for mixtures of enantiomers (the majority-rules effect), and quantitatively account for how this affects the thermodynamic stability of the assemblies. Our method allows for a direct comparison with experimental data, providing an unambiguous determination of the key parameters of the model, i.e., the mismatch and the helix reversal penalties. We demonstrate the successful application of this model to calorimetry data for bis-urea-based helical nanotubes, showing that reversals in the handedness of these nanotubes are not all that rare even though the helix reversal penalty is fairly large. By contrast, the mismatch penalty we obtain is small, implying that a large proportion of enantiomers are present in tube fractions not of their preferred handedness.
\end{abstract}

KEYWORDS . chirality; majority-rule; self-assembly; nanotube.

\section{Introduction}

Amplification of chirality, where a small imbalance in a chiral constituent is propagated into a strong optical purity, is of utmost importance in fields such as catalysis, liquid crystals and our understanding of the origin of chirality in biomolecules. ${ }^{1-8}$ While chiral amplification may be expressed at a covalent level, i.e., by transfer of the chirality of a reactant to a product, it may also be supramolecular by propagation of a chiral imbalance in composition into a helical packing of these molecules. In particular, chiral amplification can occur in self-assembled helical stacks of molecules called supramolecular polymers that are often stabilized by hydrogen bonding and/or $\pi$-stacking. ${ }^{5,-12}$ These systems are of great interest, because their dynamic nature allows them to spontaneously reach a thermodynamically stable state implying that chirality amplification can be investigated over a wide composition space by a simple mixing of constituents, e.g., chiral and achiral compounds or mixtures of enantiomers. ${ }^{13-24}$ 
In this context, two chiral amplification effects have been described: the so-called "sergeants-andsoldiers" principle involving mixtures of chiral and achiral units, and the "majority-rules" principle in mixtures of enantiomers. The "sergeants-and-soldiers" principle implies a control of the helicity of a large number of achiral units (the soldiers) by a few chiral units (the sergeants), whereas in the "majority-rules" principle a slight excess of one enantiomer leads to a strong bias towards the helicity of that enantiomer. A complete understanding of these phenomena and their possible extension to more complex systems ${ }^{25-27}$ requires their full characterization and a quantitative description of the energetics involved.

Up to now, two models have been proposed that succesfully describe available experimental data. About ten years ago, van Gestel and co-workers showed that chiral amplification in polymer-like dynamic assemblies can be rationalized by considering two free energies: a helix reversal penalty (HRP) penalizes reversals in the helical screw sense along the chain, and a mismatch penalty (MMP) arises when a monomer is present in a helix of its non-preferred screw sense. ${ }^{28,29}$ For small values of the MMP relative to the thermal energy, equivalent to about $2.5 \mathrm{~kJ} / \mathrm{mole}$ at room temperature, both enantiomers co-assemble instead of separating into enantiopure assemblies, while a large HRP value ensures that a given helical sense is propagated over long stretches along the stacks. Therefore, when both conditions are met (a low MMP and a large HRP), chiral amplification occurs. This particularly simple model has been shown to accurately describe a wide range of experimental data. ${ }^{30-34}$ In a slightly different approach, Markvoort et al. have recently shown that when enantiomers assemble through stepwise additions and dissociations of single monomers from the ends of the supramolecular polymers, that is, when scission and recombination of the chains can be neglected, then chiral amplification occurs if the cooperativity of the growth of the supramolecular polymer is strong and the MMP is low. ${ }^{35,36}$ Therefore, by construction this model rules out the occurrence of helix reversals, which is reasonable as long as the assemblies are short on a scale set by the helix reversal penalty. ${ }^{37}$ Whether assemblies are short or long on this scale in turn depends on the concentration of monomers in solution, the binding strength of identical enantiomers in an assembly and the level of cooperativity of the supramolecular polymerization. So, this model also hinges on two free energies: strong chiral amplification requires a low MMP and a high cooperativity.

As far as experimental studies are concerned, e.g., aiming to probe these two free energies, they invariantly rely on chiroptical spectroscopy. This is obviously a very powerful tool for probing chirality in supramolecular assemblies, but has some significant limitations. Indeed, circular dichroïsm (CD) spectrocopy can only be used when (i) the compounds studied possess a suitable chromophore, (ii) the solutions involve non-absorbing solvents, and (iii) the concentration is in a range limited by the sensitivity and the saturation of the detectors. In fact, it would be of interest to be able to use additionnal techniques that do not necessarily report on the chirality of the assemblies but that characterize their thermodynamic stability. Indeed, mismatches and helix reversals, for instance, can be seen as defects that arise because of thermal fluctuations (i.e., entropy), and their presence directly affects the energetics and hence stability of the assemblies. Therefore, probing the equilibrium between the assembly and the monomer state or between competing assembled states provides information on the energetics of chiral amplification. In principle, this can be done by spectroscopic (NMR, FTIR, fluorescence) and/or calorimetric (DSC, ITC) techniques, as long as a model is available to obtain (in this case) the mismatch and helix reversal penalties from the thermodynamic data.

Here we establish mismatch and helix reversal penalties in tubular supramolecular assemblies of mixtures of chiral bisureas in cyclohexane. We are able to fix these free energies unambiguously from the van Gestel model, ${ }^{29,30}$ because the self-assembled bisurea nanotubes undergo a sharp transition to a non-chiral filamentous state. The transition temperature we pinpoint by calorimetry, and make use of the weak but measurable dependence of this temperature on the enantiomeric excess. The shift of the transition temperature with enantiomeric excess can be linked directly to the energetics and statistics of the helix reversals and mismatches in the chiral nanotubes. We use the principle of the supramolecular balance for that purpose, a principle that has proven extremely sensitive to small energetic differences 
in assembled states. ${ }^{38,39}$ We find for that in our system of chiral tubes helix reversals do occur, and obtain for the HRP a value of about $19.5 \mathrm{~kJ} /$ mole whilst the MMP turns out extremely small and of the order of $0.05 \mathrm{~kJ} / \mathrm{mole}$.

In the following, we shall first discuss the ingredients of the model after which we confront the model predictions with our experimental data. We end this paper with a discussion and conclusions.

\section{Model}

For a detailed discussion of the van Gestel model we refer to the original works, ${ }^{28,29}$ and suffice with presenting the main ingredients. Starting point is a dilute solution of long aggregates formed by a mixture of two enantiomers. To describe the conformational state of the aggregates, we apply a onedimensional two-state (Ising) model. The chiral monomer that has a preferred left-handed (or righthanded) screw sense we abbreviate as the "_" monomer (or the "+" monomer). These monomers can be linked in two ways: one corresponding to a right-handed helical conformation and one corresponding to a left-handed one. These ways of bonding we refer to as the "+" bond, which is the bond type most compatible with the "+" monomer, and the "-" bond, which is most compatible with the "_" monomer. The HRP penalizes a helix reversal along the chain, and the MMP penalizes a mismatch between the preferred screw sense of a monomer and the bonds near to it. From the Hamiltonian of the system, van Gestel et al. derived formal expressions for the enantiomeric excess $(x)$ and the net helicity $(\eta)$ as a function HRP, MMP and the chemical potential difference of the two components. ${ }^{28,29}$

According to van Gestel et al., ${ }^{29}$ the enantiomeric excess $x$ can be expressed as:

$$
x=-1+\left.2 \frac{z}{\lambda_{+}} \frac{\partial \lambda_{+}}{\partial z}\right|_{s=1},
$$

where

$$
\left.\lambda_{+}\right|_{s=1}=\frac{1}{2}(1+z+z w+w+\sqrt{\alpha})
$$

is the largest eigenvalue of the transfer matrix of the one-dimensional model Hamiltonian that accounts for the presence of two kinds of monomer and two kinds of bonded interaction between pairs of monomer, that is, left- and right-handed helical. Here, $z$ is the excess fugacity of the one of the enantiomers over the other. It acts as a dummy variable that we in a later stage get rid of by connecting it to the enantiomeric excess. The quantity $s$ that enters in eq 1 in the subscript, and also below in other expressions, describes the strength of the left-handed and right-handed bonded configuration. We set it equal to unity in our description here and below, implying that they are presumed equally strong. $w=e^{-W}$ is Boltzmann weight associated with the mismatch free energy $W$ (MMP) in units of thermal energy $k_{B} T$ where $k_{B}$ is Boltzmann's constant and $T$ the absolute temperature.

Finally, we have

$$
\alpha=(z w+1-w-z)^{2}+4 \sigma(z w+1)(w+z),
$$

and

$$
\left.\frac{\partial \lambda_{+}}{\partial z}\right|_{s=1}=\frac{1}{2}\left(w+1+\frac{1}{2} \frac{1}{\sqrt{\alpha}} \frac{\partial \alpha}{\partial z}\right),
$$

where

$$
\frac{\partial \alpha}{\partial z}=2(w+z)(1-w+2 w \sigma)+2(z w+1)(2 \sigma+w+1)
$$

with $\sigma=e^{-2 R}$ the Boltzmann weight associated with the helix reversal penalty $R$ (HRP), also in units of the thermal energy.

Analogously, the net helicity $\eta$, defined as the difference between the relative number of left- and right-handed helical bonds, can be expressed as:

$$
\eta=-1+\left.2 \frac{1}{\lambda_{+}} \frac{\partial \lambda_{+}}{\partial s}\right|_{s=1},
$$


where

$$
\left.\frac{\partial \lambda_{+}}{\partial s}\right|_{s=1}=\frac{1}{2}\left(w+z+\frac{1}{2} \frac{1}{\sqrt{\alpha}} \frac{\partial \alpha}{\partial s}\right),
$$

and

$$
\frac{\partial \alpha}{\partial s}=2(w+z)((2 \sigma-1)(z w+1)+w+z) \text {. }
$$

Solving eqs 1 and 2 for $z$ produces a functional dependence of the helicity $\eta$ and the enantiomeric excess $x$, which are functions of $w$ and $\sigma$. It is this functional dependence, which can be evaluated numerically, that we use to do our curve fitting.

In principle, adjusting the values of the two model parameters allows us to fit experimental CD data and therefore yields the energetic parameters of the system. Unfortunately, in many situations, more than one pair of values produce a good fit of a given set of data. ${ }^{31,34}$ Indeed, the net helicity is not a sensitive function of the HRP, making it difficult to determine this free energy with precision. For example, Figure 1a shows two pairs of parameter values that yield very similar curves (see also Figure S1a). Discriminating between those two pairs of values based on CD data alone would require experimental data with an exquisitely low noise level.

However, the van Gestel model can be exploited further to extract information on the thermodynamic stability of the aggregates. Although not made explicit in reference 29, the average mismatch fraction and the average fraction helix reversals can be calculated straightforwardly from the model. First, from standard statistical mechanical theory, the mismatch fraction $m$ can be obtained from:

$$
m=\left.\frac{w}{\lambda_{+}} \frac{\partial \lambda_{+}}{\partial w}\right|_{s=1},
$$

where

$$
\left.\frac{\partial \lambda_{+}}{\partial w}\right|_{s=1}=\frac{1}{2}\left(z+1+\frac{1}{2} \frac{1}{\sqrt{\alpha}} \frac{\partial \alpha}{\partial w}\right),
$$

and

$$
\frac{\partial \alpha}{\partial w}=2(w+z)(2 z \sigma-z+1)+2(z w+1)(2 \sigma+z-1) .
$$

Analogously, the helix reversal fraction $h$ can be obtained from:

$$
h=\left.\frac{\sqrt{\sigma}}{\lambda_{+}} \frac{\partial \lambda_{+}}{\partial \sqrt{\sigma}}\right|_{s=1},
$$

where

$$
\left.\frac{\partial \lambda_{+}}{\partial \sqrt{\sigma}}\right|_{s=1}=\frac{1}{4} \frac{1}{\sqrt{\alpha}} \frac{\partial \alpha}{\partial \sqrt{\sigma}},
$$

and

$$
\frac{\partial \alpha}{\partial \sqrt{\sigma}}=8(z w+1)(w+z) \sqrt{\sigma} .
$$

The overall destabilization of the assemblies due to the presence of mismatches and helix reversals is then described by the total (free) energy penalty associated with these defects,

$$
\Delta E_{\text {defect }}=\Delta E_{\text {helix reversal }}+\Delta E_{\text {mismatch }}=h \cdot W+m \cdot R \text {. }
$$

Finally, inserting the value for $z$ (obtained by numerically solving eq 2 ) into eqs 3 and 4 and then into eq 5, allows us to plot the energetic penalty due to the defects versus the enantiomeric excess for arbitrary values of $W$ and $R$, that is, of the MMP and HRP. Figures $1 \mathrm{~b}, \mathrm{c}$ and $\mathrm{S} 1 \mathrm{~b}, \mathrm{c}$ shows the resulting curves for the same parameter sets as before. Although the defect energy curves in Figures $1 \mathrm{~b}$ and $1 \mathrm{c}$ 
have similar shapes, their magnitudes differ by one order of magnitude. Therefore, the two sets of values for HRP and MMP yield very similar helicities (Figure 1a), but clearly distinct energetics (Figures $1 \mathrm{~b}$ and 1c). Such large differences produce measurable differences in the transition temperature of the nanotubes to the filamentous state. Nanotube stability data are by implication much more discriminating than helicity data, and ideally one would want to rely on both. Such relative stability data versus enantiomeric composition can easily be acquired by standard spectroscopic or calorimetric techniques. As shown here, this approach actually yields more precise values for HRP and MMP.
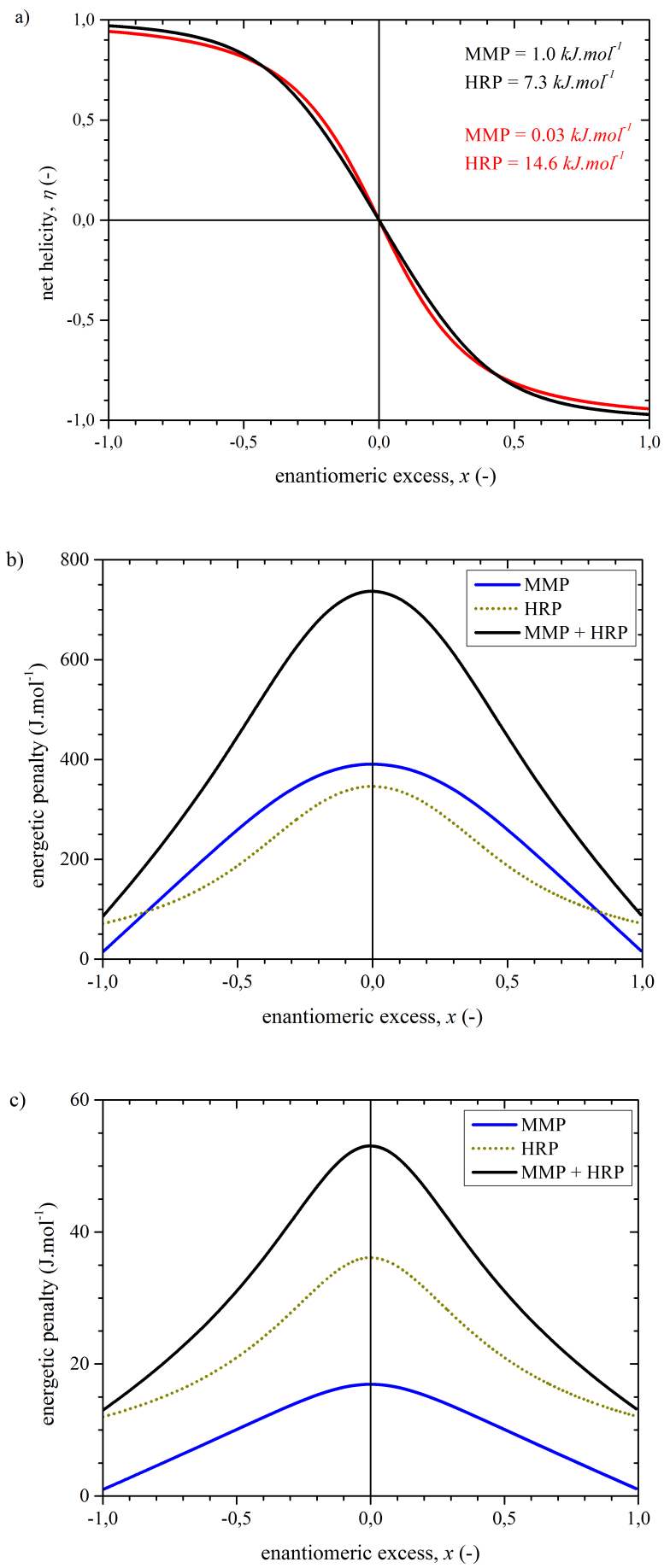

Figure 1. Net helicity as a function of enantiomeric excess (ee) from the van Gestel model for two pairs of values of the mismatch and helix reversal penalties (a). Mismatch penalty ( $\Delta E_{\text {mismatch }}$ in blue), helix 
reversal penalty ( $\Delta E_{\text {helix reversal }}$ in green) and their sum ( $\Delta E_{\text {defect }}$ in black) (b and c). The calculations were performed with the following parameters MMP $=1.0 \mathrm{~kJ} \cdot \mathrm{mol}^{-1}$ and HRP $=7.3 \mathrm{~kJ} \cdot \mathrm{mol}^{-1}:(\mathrm{a}$, black curve) and (b) or MMP $=0.03 \mathrm{~kJ} \cdot \mathrm{mol}^{-1}$ and HRP $=14.6 \mathrm{~kJ} \cdot \mathrm{mol}^{-1}:(\mathrm{a}$, red curve) and (c).

\section{Confrontation with experiment}

We now test this approach on our experimental system: in non polar solvents, bisureas EHUT-SS and EHUT-RR (Chart 1) have been shown to self-assemble by hydrogen bonding into very long nanotubes at low temperatures and into filaments at higher temperatures. ${ }^{40-42}$ The nanotubes display a very strong majority-rules effect (Figure 2a), but the helicity data are such that they could not allow for a precise determination of the energetic penalties. Only the following limits could be proposed: HRP $\geq 17 \mathrm{~kJ} / \mathrm{mol}$ and MMP $\leq 0.1 \mathrm{~kJ} / \mathrm{mol}^{34}$ Unlike the nanotubes, the filaments are CD silent so that their stability can be assumed to be independent of the enantiomeric composition. The transition between the helical nanotubes and the non-helical filaments is very cooperative and can be detected by a differential scanning calorimetry experiment. ${ }^{43}$ Actually, the value of this transition is a direct measure of the nanotube stability compared to that of the filament. ${ }^{38}$ We thus measured the nanotube to filament transition temperature versus the enantiomeric excess of bis-urea solutions (Figure S4 and Table S1) and converted these values onto an energy scale (Figure $2 b$ ) by using the aforementioned supramolecular balance concept. ${ }^{38} \mathrm{~A}$ simultaneous fit of both the helicity and stability data according to the elaborated theory of van Gestel presented above (Figure 2), yields the following values for the helix reversal penalty $\mathrm{HRP}=19.5 \mathrm{~kJ} / \mathrm{mol}$ and the mismatch penalty $\mathrm{MMP}=0.05 \mathrm{~kJ} / \mathrm{mol}^{4}{ }^{4}$

Now, this refined analysis affords a better description of the thermodynamics of the chiral amplification of bis-urea nanotubes. In particular, it is possible to visualize the amount of defects in the assemblies (Figure 2c). For example, in the racemic mixture, slightly less than half the monomers (49\%) are mismatched, which is only possible because the MMP is so low. In fact, this high content of mismatches is responsible for most of the energetic cost due to defects. The fraction of helix reversals $\left(3.31^{-4}\right)$ is much lower but not negligible: it corresponds to one helix reversal every 3000 units. To check the consistency of this approach, it is useful to compare this last value to the degree of polymerization of the tubes. We presently have no direct measurement in cyclohexane, but in toluene the degree of polymerization is 7400 (at $1 \mathrm{mM}$ and $20^{\circ} \mathrm{C}$ ). ${ }^{43,45} \mathrm{In}$ cyclohexane, the degree of polymerization is expected to be much larger than in toluene because (i) cyclohexane is less polar than toluene, and (ii) the crossover temperature from filament to nanotubes is higher in cyclohexane (51 versus $43^{\circ} \mathrm{C}^{40}$ ). Therefore, the coherence length of the helices must be significantly shorter than the length of the nanotubes: although rare events, helix reversals occur several times along each nanotube. It shows that the assumption of Markvoort et al. ${ }^{35}$ of zero helix reversals does not apply to our system.

Chart 1. Structure of bis-ureas.

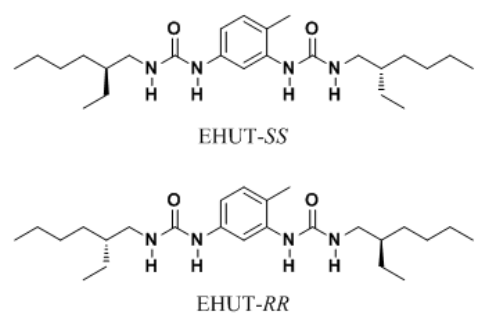


a)
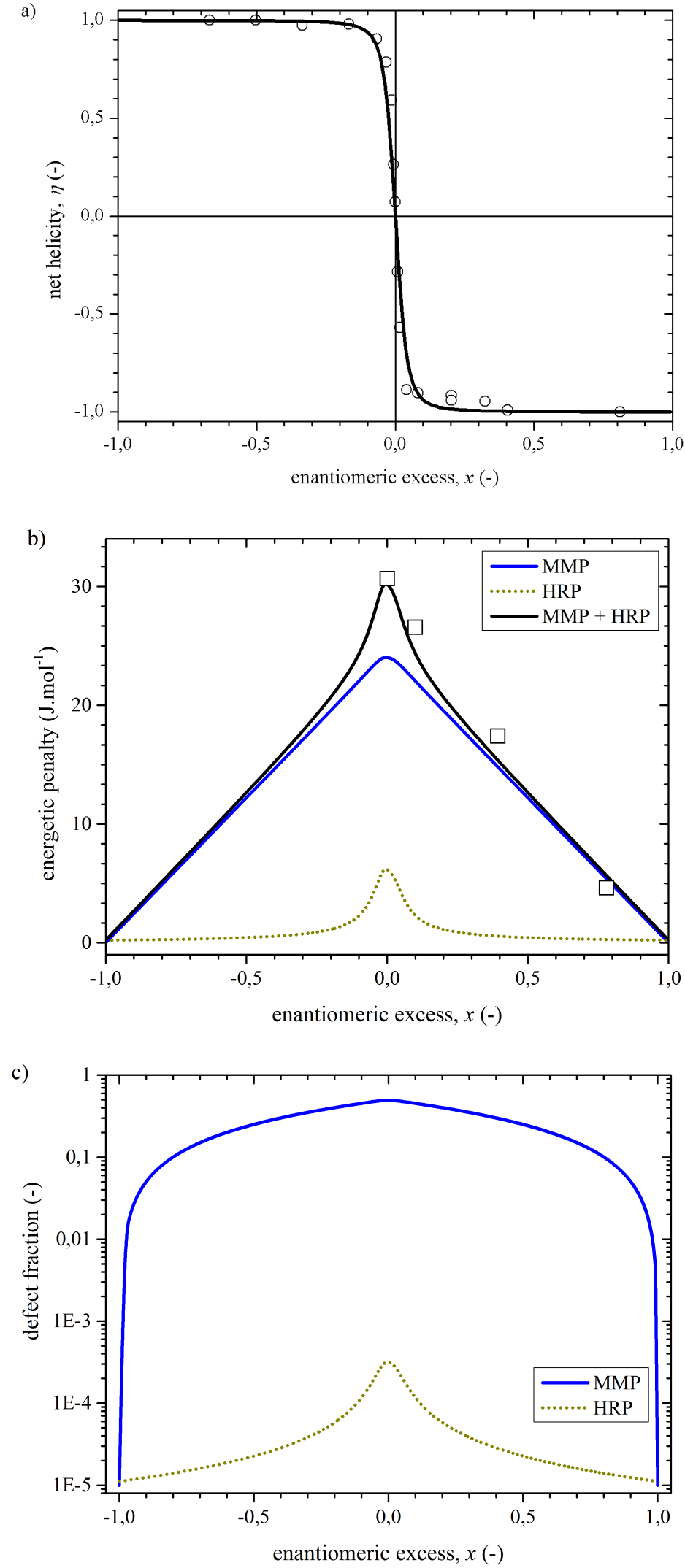

Figure 2. Net helicity (a), energetic penalty (b), and fraction of defects (c) as a function of the enantiomeric excess. Lines are theoretical predictions based on simultaneously fitting to the experimental values for the net helicity and energetic penalty, indicated by the symbols. The energetic penalty was calculated with eqs 1 to 5 , and then shifted vertically to take $x=1$ as the reference. 


\section{Conclusion}

In conclusion, we have applied and extended van Gestel's model that describes the chirality amplification in helical supramolecular polymers. It quantitatively accounts for the highly non-linear evolution of helicity versus enantiomeric excess for mixtures of enantiomers (the majority-rules effect). The extended model now describes the thermodynamic stability of self-assembled nanotubes relative to the filamentous state of bisureas and allows for a direct comparison with our experimental data. Taking into account the influence of the enantiomeric excess on the stability of the assemblies yields an unambiguous determination of the key parameters of the model, i.e., the mismatch and the helix reversal penalties. CD spectroscopy is of course required to detect chirality effects, but our approach now opens up avenues to study chiral amplification with other experimental techniques, thus extending the range of experimental conditions accessible for such studies.

Supporting Information Available. Experimental procedures and implementation of the theoretical model (Microsoft Excel file). This material is available free of charge via the Internet at http://pubs.acs.org.

\section{REFERENCES.}

(1) Todd, M. H. Asymmetric autocatalysis: product recruitment for the increase in the chiral environment. Chem. Soc. Rev. 2002, 31, 211-222.

(2) Soai, K.; Kawasaki, T. Discovery of Asymmetric Autocatalysis with Amplification of Chirality and Its Implication in Chiral Homogeneity of Biomolecules. Chirality 2006, 18, 469-478.

(3) Eelkema, R.; Feringa, B. L. Amplification of chirality in liquid crystals. Org. Biomol. Chem. 2006, 4, 3729-3745.

(4) Maeda, K.; Yashima, E. Dynamic Helical Structures: Detection and Amplification of Chirality. Top. Curr. Chem. 2006, 265, 47-88.

(5) Palmans, A. R. A.; Meijer, E. W. Amplification of Chirality in Dynamic Supramolecular Aggregates. Angew. Chem.Int.Ed. 2007, 46, 8948-8968.

(6) Satyanarayana, T.; Abraham, S.; Kagan, H. B. Nonlinear Effects in Asymmetric Catalysis. Angew. Chem. Int. Ed. 2009, 48, 456-494.

(7) Ernst, K.-H. Amplification of Chirality at Solid Surfaces. Orig. Life Evol. Biosph. 2010, 40, 4150 .

(8) Jain, V.; Cheon, K.-S.; Tang, K.; Jha, S.; Green, M. M. Chiral Cooperativity in Helical Polymers. Isr. J. Chem. 2011, 51, 1067-1074.

(9) Lohr, A.; Würthner, F. Evolution of Homochiral Helical Dye Assemblies: Involvement of Autocatalysis in the "Majority-Rules" Effect. Angew. Chem., Int. Ed. 2008, 47, 1232-1236.

(10) Lohr, A.; Würthner, F. Chiral Amplification, Kinetic Pathways, and Morphogenesis of Helical Nanorods upon Self-assembly of Dipolar Merocyanine Dyes. Isr. J. Chem. 2011, 51, 1052-1066.

(11) Rosen, B. M.; Peterca, M.; Morimitsu, K.; Dulcey, A. E.; Leowanawat, P.; Resmerita, A.-M.; Imam, M. R.; Percec, V. Programming the Supramolecular Helical Polymerization of Dendritic Dipeptides via the Stereochemical Information of the Dipeptide. J. Am. Chem. Soc. 2011, 133, 5135515. 
(12) Garcia, F.; Sanchez, L. Structural Rules for the Chiral Supramolecular Organization of OPEbased Discotics: Induction of Helicity and Amplification of Chirality. J. Am. Chem. Soc. 2012, 134, 734-742.

(13) Hirschberg, J. H.; Brunsveld, L.; Ramzi, A.; Vekemans, J. A.; Sijbesma, R. P.; Meijer, E. W. Helical self-assembled polymers from cooperative stacking of hydrogen-bonded pairs. Nature 2000, 407, 167-170.

(14) Fechtenkötter, A.; Tchebotareva, N.; Watson, M.; Müllen, K. Discotic Liquid Crystalline Hexabenzocoronenes Carrying Chiral and Racemic Branched Alkyl Chains: Supramolecular Engineering and Improved Synthetic Methods. Tetrahedron 2001, 57, 3769-3783.

(15) Fenniri, H.; Deng, B.-L.; Ribbe, A. E. Helical Rosette Nanotubes with Tunable Chiroptical Properties. J. Am. Chem. Soc. 2002, 124, 11064-11072.

(16) Bushey, M. L.; Hwang, A.; Stephens, P. W.; Nuckolls C. The Consequences of Chirality in Crowded Arenes-Macromolecular Helicity, Hierarchical Ordering, and Directed Assembly. Angew. Chem. Int. Ed. 2002, 41, 2828-2831.

(17) Hirschberg, J. H. K. K.; Koevoets, R. A.; Sijbesma, R. P.; Meijer, E. W. Helical Supramolecular Aggregates Based on Ureidopyrimidinone Quadruple Hydrogen Bonding. Chem. Eur. J. 2003, 9, 42224231 .

(18) Jin, W.; Fukushima, T.; Niki, M.; Kosaka, A.; Ishii, N.; Aida T. Self-assembled Graphitic Nanotubes with one-handed Helical Arrays of a Chiral Amphiphilic Molecular Graphene. Proc. Natl. Acad. Sci. USA 2005, 102, 10801-10806.

(19) Mateos-Timoneda, M. A.; Crego-Calama, M.; Reinhoudt, D. N. Controlling the Amplification of Chirality in Hydrogen-bonded Assemblies. Supramol. Chem. 2005, 17, 67-79.

(20) Ishi-i, T.; Kuwahara, R.; Takata, A.; Jeong, Y.; Sakurai, K.; Mataka, S. An Enantiomeric Nanoscale Architecture Obtained from a Pseudoenantiomeric Aggregate: Covalent Fixation of Helical Chirality Formed in Self-Assembled Discotic Triazine Triamides by Chiral Amplification. Chem. Eur. J. 2006, 12, 763-776.

(21) Shikata, T.; Kuruma, Y.; Sakamoto, A.; Hanabusa, K. Segment Sizes of Supramolecular Polymers of N,N',N"'-Tris(3,7-dimethyloctyl)benzene-1,3,5-tricarboxamide in n-Decane. J. Phys. Chem. B 2008, 112, 16393-16402.

(22) Danila, I.; Riobé, F.; Piron, F.; Puigmarti-Luis, J.; Wallis, J. D.; Linares, M.; Ågren, H.; Beljonne, D.; Amabilino, D. B.; Avarvari N. Hierarchical Chiral Expression from the Nano- to Mesoscale in Synthetic Supramolecular Helical Fibers of a Nonamphiphilic C3-Symmetrical $\pi$ Functional Molecule. J. Am. Chem. Soc. 2011, 133, 8344-8353.

(23) Aparicio, F.; Matesanz, E.; Sanchez, L. Cooperative Self-Assembly of Linear Organogelators. Amplification of Chirality and Crystal Growth of Pharmaceutical Ingredients. Chem. Commun. 2012, $48,5757-5759$.

(24) Aparicio, F.; Garcia, F.; Sanchez, L. Supramolecular Polymerization of $\mathrm{C}_{3}$-Symmetric Organogelators: Cooperativity, Solvent, and Gelation Relationship. Chem. Eur. J. 2013, 19, 3239-3248. 
(25) Anderson, T. W.; Sanders, J. K. M.; Pantos, G. D. The sergeants-and-soldiers effect: chiral amplification in naphthalenediimide nanotubes. Org. Biomol. Chem. 2010, 8, 4274-4280.

(26) Helmich, F.; Smulders, M. M. J.; Lee, C. C.; Schenning, A. P. H. J.; Meijer, E. W. Effect of Stereogenic Centers on the Self-Sorting, Depolymerization, and Atropisomerization Kinetics of Porphyrin-Based Aggregates. J. Am. Chem. Soc. 2011, 133, 12238-12246.

(27) Cantekin, S.; ten Eikelder, H. M. M.; Markvoort, A. J.; Veld, M. A. J.; Korevaar, P. A.; Green, M. M.; Palmans, A. R. A.; Meijer, E. W. Consequences of Cooperativity in Racemizing Supramolecular Systems. Angew. Chem. Int. Ed. 2012, 51, 6426-6431.

(28) van Gestel, J.; van der Schoot, P.; Michels, M. A. J. Amplification of Chirality in Helical Supramolecular Polymers. Macromolecules 2003, 36, 6668-6673.

(29) van Gestel, J. Amplification of Chirality in Helical Supramolecular Polymers: The MajorityRules Principle. Macromolecules 2004, 37, 3894-3898; Macromolecules 2006, 39, 1664.

(30) van Gestel, J.; Palmans, A. R. A.; Titulaer, B.; Vekemans, J. A. J. M.; Meijer, E. W. "MajorityRules" Operative in Chiral Columnar Stacks of C3-Symmetrical Molecules. J. Am. Chem. Soc. 2005, $127,5490-5494$.

(31) Smulders, M. M. J.; Filot, I. A. W.; Leenders, J. M. A.; van der Schoot, P.; Palmans, A. R. A.; Schenning, A. P. H. J.; Meijer, E. W. Tuning the Extent of Chiral Amplification by Temperature in a Dynamic Supramolecular Polymer. J. Am. Chem. Soc. 2010, 132, 611-619.

(32) Smulders, M. M. J.; Stals, P. J. M.; Mes, T.; Paffen, T. F. E.; Schenning, A. P. H. J.; Palmans, A. R. A.; Meijer, E. W. Probing the Limits of the Majority-Rules Principle in a Dynamic Supramolecular Polymer. J. Am. Chem. Soc. 2010, 132, 620-626.

(33) Cantekin, S.; Balkenende, D. W. R.; Smulders, M. M. J.; Palmans, A. R. A.; Meijer, E. W. The Effect of Isotopic Substitution on the Chirality of a Self-assembled Helix. Nat. Chem. 2011, 3, 42-46.

(34) Isare, B.; Linares, M.; Zargarian, L.; Fermandjian, S.; Miura, M.; Motohashi, S.; Vanthuyne, N.; Lazzaroni, R.; Bouteiller, L. Chirality in Dynamic Supramolecular Nanotubes Induced by a Chiral Solvent. Chem. Eur. J. 2010, 16, 173-177.

(35) Markvoort, A. J.; ten Eikelder, H. M. M.; Hilbers, P. A. J.; de Greef, T. F. A.; Meijer, E. W. Theoretical Models of Nonlinear Effects in two-component Cooperative Supramolecular Copolymerizations. Nat. Commun. 2011, 2, 509.

(36) Ten Eikelder, H. M. M.; Markvoort, A. J.; de Greef, T. F. A.; Hilbers, P. A. J. An Equilibrium Model for Chiral Amplification in Supramolecular Polymers. J. Phys. Chem. B 2012, 116, 5291-5301.

(37) Jabbari-Farouji, S.; van der Schoot, P. Theory of Supramolecular Co-polymerization in a twoComponent System. J. Chem. Phys. 2012, 137, 064906

(38) Roman, M.; Cannizzo, C.; Pinault, T.; Isare, B.; Andrioletti, B.; van der Schoot, P.; Bouteiller, L. Supramolecular Balance: Using Cooperativity to Amplify Weak Interactions. J. Am. Chem. Soc. 2010, 132, 16818-16824.

(39) Bouteiller, L.; van der Schoot, P. Probing Intermolecular Interactions in Self-Assembled Nanotubes. J. Am. Chem. Soc. 2012, 134, 1363-1366. 
(40) Bouteiller, L.; Colombani, O.; Lortie, F.; Terech, P. Thickness Transition of a Rigid Supramolecular Polymer. J. Am. Chem. Soc. 2005, 127, 8893-8898.

(41) Pinault, T.; Isare, B.; Bouteiller, L. Solvents with Similar Bulk Properties Induce Distinct Supramolecular Architectures. Chem. Phys. Chem. 2006, 7, 816-819.

(42) Shikata, T.; Nishida, T.; Isare, B.; Linares, M.; Lazzaroni, R.; Bouteiller, L. Structure and Dynamics of a Bisurea Based Supramolecular Polymer in n-Dodecane. J. Phys. Chem. B 2008, 112, 8459-8465.

(43) Bellot, M.; Bouteiller, L. Thermodynamic Description of Bis-Urea Self-Assembly: Competition Between Two Supramolecular Polymers. Langmuir 2008, 24, 14176-14182.

(44) The robustness of the method is illustrated by Figures S2 and S3 that show several simulated curves corresponding to other values of HRP and MMP.

(45) Pinault, T.; Cannizzo, C.; Andrioletti, B.; Ducouret, G.; Lequeux, F.; Bouteiller, L. Anions as Efficient Chain Stoppers for Hydrogen Bonded Supramolecular Polymers. Langmuir 2009, 25, 84048407. 